\title{
Le kelpie, ou les monstres lacustres
}

Jean Berton

\section{(2) OpenEdition}

\section{Journals}

\section{Édition électronique}

URL : http://journals.openedition.org/etudesecossaises/3461

DOI : 10.4000/etudesecossaises.3461

ISSN : 1969-6337

\section{Éditeur}

UGA Éditions/Université Grenoble Alpes

\section{Édition imprimée}

Date de publication : 1 mars 2001

Pagination : 151-173

ISBN : 978-2-84310-198-4

ISSN : $1240-1439$

Référence électronique

Jean Berton, «Le kelpie, ou les monstres lacustres », Études écossaises [En ligne], 7 | 2001, mis en ligne le, consulté le 08 septembre 2020. URL : http://journals.openedition.org/etudesecossaises/3461 ;

DOI : https://doi.org/10.4000/etudesecossaises.3461 


\section{Le kelpie, ou les monstres lacustres}

JEAN BERTON

Université de Saint-Étienne.

1. The Monarch of the Glen, Hunting the Fairies, Keep the Home Guard Turning, et Whisky Galore.
Dans le quotidien glaswégien, The Herald, daté du 2 septembre 2000, p. 12, Cameron Simpson introduit sa recension de The Encyclopedia of the Loch Ness Monster, de Paul Harrison, par une citation de J. F. Kennedy: The great enemy of truth is very often not the lie but the myth - persistent, persuasive, and unrealistic. Cette citation aurait fort bien pu servir pour présenter le roman de Compton Mackenzie, The Rival Monster. Cette comédie de mœurs, publiée en 1952, fait partie d'une série de romans ${ }^{1}$ dont l'action se situe dans deux îles voisines et imaginaires, Great Todday et Little Todday, elles-mêmes situées au cœur de l'archipel des Hébrides. L'objectif de l'auteur est moins de fustiger ou de corriger les travers de cette société isolée que de distraire le lecteur aux dépens d'une communauté réduite au rôle d'échantillon représentatif de l'archipel, voire des Hautes Terres. Dans ces romans se trouvent tous les ingrédients nécessaires à la composition d'une bonne comédie écossaise : le whisky, la religion, l'Anglais, la romance...

Le but de cette étude est d'analyser non pas les éléments comiques du roman, The Rival Monster, mais les tenants et les aboutissants de son "primum mobile» distinctif: le monstre lacustre, devenu veuf, aurait quitté le Loch Ness pour venir hanter les rivages des Îles Todday à la recherche de l'âme sœur. Ce monstre-des-eaux, tantôt lacustre, tantôt marin, est-il le pur produit d'une imagination sous l'emprise de spiritueux ou bien l'avatar de créatures de légendes picto-celtes synthétisées en mythe? Mackenzie se garde bien de suggérer que son Nessie est le reflet d'une certaine réalité, mais laisse son lecteur inférer que si Great Todday et Little Todday sont vraisemblablement à l'image des îles de l'archipel des Hébrides, Nessie doit être l'image d'une créature réelle. Si nous cherchions à provoquer l'ire du plus conservateur des personnages du roman, le chef de clan Donald MacDonald of Ben Nevis, propriétaire du Loch Ness et anti-bolchévique notoire, nous pourrions avancer, dans une lecture marxisante, que Nessie, ou son double, est 
asservi aux désirs d'alimenter la chronique d'un district hébridien. En effet, l'action se situant au lendemain de la seconde guerre mondiale, C. Mackenzie fait intervenir, dans le premier chapitre, des chauffards extraterrestres dont le méfait tragique pousserait Nessie, à moins que ce ne soit sa compagne (sa moitié ou son double, sa commère ou son alter ego... les qualificatifs révélateurs de schizophrénie sont multiples), à venir hanter la zone de ses îles imaginaires.

\section{Une fiction}

Compton Mackenzie a montré dans sa série de romans qu'il sait utiliser à son profit tout pseudo-événement socio-historique, tel que le naufrage d'un navire au cours de la seconde guerre mondiale dans Whisky Galore. Ainsi s'est-il emparé d'un phénomène étrange qui générait un grand tapage médiatique depuis 1933, soit moins de vingt ans au moment de la composition du roman. L'auteur se garde bien de prendre partie dans la polémique, mais il se révèle bien informé des caractéristiques du phénomène qu'il distribue au gré des chapitres pour assurer la dynamique du roman. The Rival Monster est une fiction qui doit être classée dans le genre comique dès le premier chapitre par l'usage de l'exagération: si, pour le narrateur, la présence de monstres lacustres dans le Loch Ness n'est ni prouvée ni réfutée, si l'existence d'objets volants non identififés était déjà source de controverse en 1950, la rencontre accidentelle dramatique d'un OVNI et d'un monstre à la surface d'un lac appartient au domaine du possible, quoique hautement improbable. Prudent, Mackenzie s'échappe de ce domaine du possible générateur de polémique en transformant la «soucoupe volante " en "théière volante», comme le précise l'unique témoin de l'accident, Mr MacLennan ${ }^{2}$ «... I would prefer to compare it to the spout of a teapot. Well, to cut a long story short, just after this flying spout passed close to where I was standing, out of the loch came something I can only describe as a huge horse's head on top of a snaky neck». Plus loin, au quatrième chapitre, l'auteur veille à confirmer le caractère exclusivement comique de son récit en décrivant le bruit du monstre aperçu dans les parages de Todday: un aboiement ou un mugissement appartiennent au domaine du possible et du connu, mais un croisement des deux sons ainsi décrit relève du comique ${ }^{3}:$ «... a cow 
that would be barking at you like a dog or a dog that would be bellowing at you like a cow and put the two noises in the foghorn of the Island Queen, you'd be having a sort of an idea what kind of a noise this crayture was after making...».

Le comique d'amplification dans le discours trouve son relais dans le récit par le biais du personnage du rédacteur en chef du Scottish Daily Tale secondé par son envoyé spécial, Carmichael. Le rôle de la presse est mis en avant dans le roman autant qu'il l'était en 1950, et les échanges épistolaires entre les lecteurs et les journalistes insérés dans la fiction reflètent la réalité quotidienne où les journalistes et les reporters donnaient une dimension nationale à un événement que les Montagnards, ou Highlanders, et les Iliens, ou Islanders, auraient souhaité conserver au niveau local. Dans le roman, l'attitude de rejet plus ou moins fort des randonneurs venus du sud est le reflet de la réalité hébridienne, de même que l'antagonisme entre Iliens écossais et Anglais. Ainsi la fiction apparaît-elle tout naturellement comme le reflet de la réalité.

Il nous faut maintenant considérer l'opposition linguistique très présente dans le roman de Mackenzie entre l'anglais et le gaélique: d'une part, le personnage de Paul Wagget est ridiculisé pour son effort dans l'apprentissage du gaélique, d'autre part, les celtophones s'expriment dans un anglais fortement influencé par la syntaxe gaélique. Mackenzie parvient aussi à traduire l'accent local sans forcer la note ${ }^{4}$ outre mesure: «"The Lord forgive you", Mr Wackett, I wass saying.... if you're after thinking it was an otter I was seeing... and if you think the crayture was an otter you'll be thinking Sahtan is the minister...». Par souci d'équité envers ses lecteurs écossais, peut-être, Mackenzie fait intervenir une Anglaise, Mrs Odd, dont l'anglais est parfois aussi approximatif que celui de la Mrs Malaprop de Sheridan ${ }^{5}$ : «a living skelington»... «What was it I sore? I saw...» «In Eddingborough». En délaissant la teneur comique du roman, nous pourrions prêter à Mackenzie l'intention de montrer indirectement au lecteur l'importance des divergences d'appréciation du problème imputables aux incompatibilités idiomatiques des langues en présence, du gaélique et de l'anglais. Après examen de ce phénomène des monstres lacustres et marins en Écosse, il s'avère que la question linguistique est un élément fondamental dans l'étude des faits. C'est

4. P. 75, passim. 5. P. 73, 80 . pourquoi il est primordial d'analyser les qualificatifs employés pour tenter d'éviter les malentendus. 
A l'instar du narrateur qui s'applique à expliciter l'étymologie des toponymes ${ }^{6}:$ «The origin of the name of Pillay is in dispute. Some Gaelic topographers say it means "the island of the winnowing"...» [...] «The origin of the name of Poppay is in dispute, but most philologists now accept it as a variant of Pabbay - the Priest's island.» nous devons analyser la terminologie en usage. Le terme le plus ambigu est celui de monstre: en effet, non seulement il admet plusieurs définitions, mais encore son emploi pour un prétendu animal aquatique entraîne l'application de l'usage de l'invariabilité. Les poissons à écailles ("salmon», «trout», etc.) ne prenant pas la marque du pluriel, cette bizarrerie grammaticale est spontanément appliquée à ces "water monster(s)", même si ces monstres n'ont pas un corps couvert d'écailles: ainsi l'expression «Loch Ness monster» est-elle ambiguë car elle peut signifier un ou plusieurs monstres. La théorie du monstre unique qui aurait survécu depuis les temps préhistoriques a la faveur des détracteurs avides de polémique, car elle permet tous les excès. En revanche, la théorie d'un "troupeau» de monstres d'un nombre non déterminé mais composé de mâles et de femelles, de vieux et de jeunes, est plus gênante car elle interpelle la science pour procéder à leur identification. Mackenzie postule la présence d'au moins deux monstres dans le Loch Ness pour servir son récit : si l'un de ces monstres a été tué dans l'accident, l'autre doit partir chercher un autre partenaire, mâle ou femelle, dans les fjords et la mer intérieure des Hébrides. L'entrée en scène d'un jeune paléontologue est un clin d'œil à l'intention des scientifiques, car les divers témoignages permettaient déjà d'orienter les recherches vers la grande famille des dinosaures: le jeune William W. Brownsworth déclare ${ }^{7}$ «I spent a fortnight last year investigating the Loch Ness Monster in the hope of being able to link it up with a plesiosaurus, an ichthyosaurus, or indeed with any of the megalosaurs». Le manque de moyens financiers est un argument, toujours d'actualité, avancé par la science officielle pour ne pas organiser une étude exhaustive de ce lac qui est le plus volumineux et le plus profond du Royaume-Uni, où pourtant des «chasseurs de monstres» ont fait des découvertes secondaires collatérales concernant la faune et la flore du lac. Ces quelque sept milliards de mètres cubes d'eau très froide et très sombre sont un

6. P. 117 et 173 . 7. P. 93. obstacle de taille qui n'a d'égal que le handicap psycho-sociologique à surmonter lorsqu'on cherche un famille d'animaux 
dont on n'est pas entièrement sûr qu'ils existent, mais qui est paradoxalement médiatisée à l'excès.

\section{Une affaire de terminologie}

Le qualificatif de monstre a été attribué par un certain Evan Barron, rédacteur en chef de l'Inverness Courier, qui, apprenant les observations faites par un de ces correspondants locaux, Alexander Campbell, garde-pêche à Fort-Augustus, se serait exclamé: «Eh bien, si l'animal est aussi gros que le dit Campbell, on ne peut pas se contenter de l'appeler une créature, ce doit être un vrai monstre». Cette déclaration péremptoire de Barron est confirmée par la publication d'un article, le 2 mai 1933, p. 5, intitulé «Strange spectacle on Loch Ness - What was it? " Cet article est devenu un repère historique majeur car il a institué le mot monstre: le terme se trouva répété par le journaliste qui utilisa tour à tour «beast», «creature», ou "monster»: «... apart from its enormous size, the beast, in taking the final plunge, sent out waves that were big enough to have been caused by a passing steamer. The watchers waited for almost half-an-hour in the hope that the monster (if such it was) would come to the surface again...». Le terme était juste puisque «monstre» convient pour qualifier un animal ou une personne de taille hors-norme ${ }^{8}$. Dans son roman, Mackenzie fait décrire la créature par le chef de clan, MacDonald of Ben Nevis, irrité par le scepticisme de son ami le Colonel Lindsay-Wolseley ${ }^{9}$ : "Yes, there's a difference [between an otter and a prehistoric monster] of anything up to eighty or ninety feet of solid flesh, which makes it much easier to have an optical delusion about an otter than about the monster". De plus, comme pour illustrer une autre acception du terme de monstre, les personnages

8. C'est une caractéristique des monstres telle que Claude Kappler les décrit dans le chapitre IV Typologie du monstre - de son ouvrage, Monstres, démons et merveilles, p. 130. Par ailleurs, au

chapitre VI-La notion de monstre-il précise, p. 219 , que «le monstre est ce qui se distingue par sa rareté», ce qui serait une autre caractéristique applicable à Nessie.

9. P. 25 . du roman qui disent avoir vu ce monstre de près font part de leur frayeur devant cet animal inconnu de grande taille, ce qui est un écho des déclarations faites par ces témoins oculaires choqués par une rencontre inopinée, sur le lac ou ses abords, avec cette chose vivante d'une laideur repoussante. C'est le sens du mot monstre tel qu'il apparaît dans la première phrase de l'article de l'Inverness Courier: «Loch Ness has for generations been credited with being the home of a fearsome-looking monster, but, somehow or other, the "water-kelpie", as this legendary creature is called, has always been regarded as a myth, if not a joke». 
Le terme de monstre est à la fois juste et trop vague pour favoriser une étude dépassionnée. Ainsi que nous le verrons plus loin, par son origine latine ${ }^{10}$, monstre peut faire référence à un être fantastique figurant dans les mythologies et les légendes.

L'antagonisme latent hérité de siècles de conflits entre l'Écosse et l'Angleterre trouva un exutoire dès que furent connues les nouvelles de manifestations peu banales à la surface du Loch Ness colportées par les ouvriers engagés à la construction de la route $\mathrm{A} 82$ destinée à relier Inverness à Fort William et Glasgow. Le terme «monstre» bientôt céda devant une appellation plus familière: Nessie. Le terme revêtait des valeurs plus affectives qui avaient l'intérêt de paraître désobligeantes parce que ce diminutif était de surcroît féminin. Plus tard, Nessie devint une mascotte en peluche que l'on pouvait offrir aux enfants. Peut-être faut-il considérer ce terme comme la forme anglicisée du terme collectif masculin en gaélique, «an Niseag». Ce nom était connu dans la région du Loch Ness depuis la nuit des temps. À ce nom était associé le malheur, car tout le monde le savait : le Loch Ness ne rend jamais ses morts, quoi qu'en ait pu prouver l'expérience. Les voyageurs depuis des siècles avaient répété que an Niseag n'était pas seul, car dans le Loch Morar, plus à l'ouest, il y avait la féroce $a$ 'Mhorag, ou dans le Loch Shiel, plus au sud, il y avait le lugubre an $t$-Shieleag. Dans les Hautes Terres, tout le monde le savait, mais il était préférable de ne pas les citer.

En revanche, on pouvait parler librement des fameux «eachuisge», comme Mackenzie dans son roman, qui signifie littéralement "water horse", et que je traduis par "cheval-des-eaux» pour englober les cas des "chevaux-des-lacs", "chevaux-desrivières», «chevaux-des-mers » et «chevaux-des-fjords » ${ }^{11}$... Le cheval-des-eaux vivait, disait-on, dans les lacs, les rivières, les fjords et la mer, et se montrait parfois à la surface. Ce n'était

10. En effet, monstrum signifie «fait prodigieux, présage, avertissement de dieux ".

11. C. Kappler présente un dessin de «Cheval de mer» aux pattes antérieures palmées et dont la partie postérieure est une longue queue terminée par un plumeau de crin. Monstres, démons et merveilles, p. 189. pas un cheval ordinaire mais à défaut d'identification on ne pouvait que l'assimiler à un cheval puisqu'il avait de toute évidence une tête de cheval et une puissance de cheval. On en voyait parfois le dos. Dans les Hautes Terres, on n'avait pas attendu l'arrivée des Romains pour connaître les chevaux ou même les taureaux à long pelage. D'ailleurs, dans les milieux aquatiques on voyait aussi des taureaux-des-eaux, appelés «tarbh-uisge» ou "water bulls», dont le comportement ne différait en rien de celui des cheveaux-des-eaux. On les distin- 
12. P. 45 .

13. Le dictionnaire Oxford English Dictionary précise que le mot est attesté depuis 1747 : «The Lowland Scottish name of a fabled spirit or demon, usually appearing in the shape of a horse, reputed to haunt lakes and rivers, and to take delight in, or bring about, the drowning of travellers and others". 14. P. 11 : Sir James Jeans and Loch Ness et Loch Ness Monster-More stories and theories.

15. P. 9:An Irish Monster Reported.

16. P. 9: Ogopogo; le Lac Okanagan est en Colombie-Britannique. guait parce que les cheveaux-des-eaux avaient souvent une crinière, et les taureaux-des-eaux avaient des cornes. Mackenzie évoque les deux animaux légendaires dans son $\operatorname{roman}^{12}$ : "[Kenny Macroon] insisted in spite of several attempts to make him compare the head he had seen emerge from the water to a horse that it was much more like that of a bull, except of course for the mane which he agreed was more equine than bovine.» Les populations soumises à la loi de l'Église depuis tant de générations avaient accepté jusqu'à la fin de l'ère victorienne la conclusion des savants qui, bien avant le Moyen Âge, avaient affirmé que le milieu aquatique hébergeait des animaux analogues à ceux que l'on voyait sur la terre ferme. D'ailleurs pourquoi serait-il absurde de croire que tous ces chevaux marins devaient brouter les algues ou le varech que l'on appelait «kelp»? Ce terme anglo-saxon a engendré un autre terme générique: le «kelpie» ${ }^{13}$.

Quand Mackenzie écrivait son roman en se prévalant de faits observés autour de lui, le nombre de naïfs crédules diminuait sensiblement; en effet, beaucoup d'iliens avaient voyagé très loin pour défendre l'Empire, comme tant d'autres avant eux. Le niveau d'instruction général égalait très certainement celui des Anglais, et pour ceux qui avaient le loisir de s'intéresser, à ces bizarreries aquatiques il importait de leur trouver un nom: les surnoms dont elles avaient été affublées jusque-là non seulement n'encourageaient pas les scientifiques à s'y intéresser mais encore excitaient les polémistes jamais avares de surenchères. Bref, leurs conclusions devenaient inéluctables: derrière chaque monstre se cachait un Écossais imbibé de whisky. C'est ainsi que la région des Hautes Terres d'Écosse toute entière s'est vite trouvée impliquée, bon gré mal gré, dans ce phénomène qui depuis le printemps 1933 prenait de l'ampleur semaine après semaine.

Qui plus est, le phénomène s'internationalisa entre le 2 mai 1933, date du célèbre article de l'Inverness Courier et le 29 décembre 1933 où The Scotsman publia quatre articles, dont deux sur le Loch Ness ${ }^{14}$ un sur l'Irlande ${ }^{15}$ et un sur le Lac Okanagan ${ }^{16}$. La question de l'identification devint urgente, plus pour des raisons politiques que scientifiques, semble-t-il, au point de faire l'objet de questions orales à la Chambre des Communes: le Hansard du 12 décembre 1933 publie la réponse de Sir Godfrey Collins. 
Mr Anstruther-Gray asked the Secretary of State for Scotland whether, in the interest of science, he will cause an investigation to be made into the existence of a monster in Loch Ness.

The Secretary of State for Scotland (Sir Godfrey Collins): There appears to be no reason to suspect the presence of any baneful monster in Loch Ness, and as regards scientific interests, I think that, in present circumstances, further researches are properly a matter for the private enterprise of scientists, aided by the zeal of the Press and of photographers.

Mr Anstruther-Gray: Will the honorary Gentleman consider inviting the assistance of the Air Department to observe and photograph this creature, so that a unique opportunity of adding to our scientific knowledge may not be thrown away?

Sir Godfrey Collins: I should like a little more evidence of the exact nature of the monster before I tried to call in the Air Force. Mr Neil Maclean: Would not the Scottish Office find their time better occupied in trying to capture the monster of unemployment in Scotland? ${ }^{17}$

La réponse de Sir Godfrey Collins a été prise pour une mise en garde à l'intention des scientifiques qui se sont toujours abstenus de venir enquêter au Loch Ness à titre officiel. Les quelques scientifiques qui ont tenté de trouver une réponse l'ont toujours fait à titre officieux et personnel et dans la plus grande discrétion.

Les gens des Hautes Terres ont appris à cultiver une attitude de réserve, sinon de mutisme, d'autant plus que, s'agissant de monstre lacustre, leur témoignage leur valait, au mieux, la réprobation pour conduite indécente. Aussi les témoins oculaires qui ne pouvaient s'empêcher de raconter ce qu'ils avaient vu finirent-ils par subir des interrogatoires serrés pour déterminer la fiabilité de leur rapport. A titre d'illustration, dans The Rival Monster, le chef de clan, le prêtre catholique de Little Todday et le rationaliste anglais de Great Todday font subir des interrogatoires à diver témoins devenus les héros du jour. Par manque de cohésion et de préparation, les interrogatoires manquaient souvent de rigueur policière, et les interrogateurs eux-mêmes ne faisaient pas toujours preuve d'impartialité scientifique.

17. La réplique agacée de Neil Maclean illustre parfaitement les priorités socio-économiques des politiques écossais, quelle que fût l'importance du tapage médiatique suscité par le phénomène Nessie.

\section{Un portrait}

Après la période de la seconde guerre mondiale, où le Loch Ness était devenu zone militaire, les Britanniques trouvèrent 
un nouvel intérêt pour Nessie et de nouvelles photos prises par des touristes ou des «chasseurs de monstre» paraissaient dans la presse. David James, député de la circonscription de formation scientifique, prit sur lui de fonder un groupe d'investigation en 1962, The Loch Ness Phenomena Investigation Bureau, afin de mener une surveillance de surface et, conjointement, de constituer une banque de données s'appuyant sur les témoignages oculaires. Les témoins devaient répondre à de multiples questions précises avant de voir leur rapport accepté ou rejeté. Ce groupe d'étude fonctionna pendant une dizaine d'années et collationna des centaines de rapports jugés recevables pour tenter de composer un portrait robot qui ne fût pas un collage d'éléments de comparaison. Les photos de surface n'étaient pas suffisamment claires mais les échos de sonars - nouveauté technologique - laissaient espérer que la chance allait tourner. Le groupe de recherche accueillit des universitaires américains qui au fil du temps parvinrent à éveiller la curiosité de quelques chercheurs du prestigieux MIT de Boston.

Le défi se révélait difficile à cause de la qualité de l'eau très froide rendue très sombre par les particules de terre de bruyère en suspension. Le matériel de surveillance subaquatique fonctionnait mal et dut être adapté saison après saison. Les difficultés d'ordre technique se doublaient de handicaps administratifs : un nouveau sonar ou une nouvelle caméra étaient retenus longtemps en douane... Les observations de surface et par sonar immergé montrant que de fréquents passages se faisaient à proximité de certaines rivières à la suite de saumons, un radeau fut construit pour soutenir une bàtterie de caméras jumelées à des projecteurs dans la baie de Urqhuart. La patience des techniciens fut récompensée pendant l'été de 1972 par une série de photos sous-marines qui montraient diverses parties de corps d'animaux non identifiés et non identifiables. La photo la plus impressionnante était celle d'une palette natatoire que les zoologistes ne purent attribuer à aucun animal connu. Sa longueur était estimée à plus d'un mètre et demi... On attendit trois ans pour qu'un symposium fût organisé à Édimbourg au mois de décembre. Mais le tapage médiatique fut tel que la réunion dut être annulée. Cependant, le groupe de chercheurs dirigés par Sir Peter Scott, parvint à organiser une réunion à Westminster le 10 décembre 1975. Non seulement on y renouvela le décret de protection de ces animaux inconnus déjà promulgué en décembre 1933, principe 
de précaution oblige, mais on inscrivit un nom scientifique: le nessiteras rhombopteryx, soit le monstre du Ness à l'aileron de forme lozangique. Ce nom scientifique, en grec, devait enfin occulter tous les surnoms dont l'espèce était affublée en attendant de pouvoir la connaître en détails. Mais les spécialistes en anagrammes eurent vite fait de transmettre à la presse leur propre interprétation: "monster hoax by Sir Peter S.».

Fort des résultats photographiques corroborant les descriptions le plus souvent partielles de ces nessiteras rhombopteryx, Sir Peter Scott dessina un portrait-robot de ce qu'il considérait comme un animal. Les réactions du monde scientifique ne furent en rien surprenantes: du haussement d'épaules à l'affirmation péremptoire d'interprétations déjà avancées et déjà réfutées, de l'indifférence déterminée à la raillerie agressive. Bref, l'animal n'existait que dans l'imagination de l'artiste... Dès 1933, on avait comparé le nessiteras rhombopteryx à un plésiosaure; puisque l'on sait que les plésiosaures ont disparu de notre planète en même temps que tous les dinosaures, le nessiteras rhombopteryx ne pouvait pas exister: CQFD! Il est vrai qu'un monstre dit préhistorique ne peut raisonnablement pas exister. L'argument est repris par Mackenzie qui fait dire à son personnage représentant l'Anglais rationaliste borné:

... I never expected to hear an M.A. of Glasgow University tell me he believed in the existence of a prehistoric monster.

I'm not prepared to say that this creature is prehistoric, Mr Wagget. We just don't know what it is, but I think we can feel quite sure that it is something nobody here has seen before.

Comparer le monstre du Loch Ness à un plésiosaure témoigne d'un esprit rationnel et informé, même si l'observa-

18. En référence à ce qu'énonce Mircea Eliade, dans Le Mythe de l'éternel retour, p. 48, "la réalité s'acquiert exclusivement par répétition ou participation", nous pouvons avancer que Nessie ne peut exister que par la présence d'autre monstres dans d'autres lacs et/ou parce qu'il répète l'archétype archéologique du plésiosaure. Une preuve supplémentaire de sa réalité populaire apparaît dans le cheval-des-eaux qui réalise la répétition de l'archétype du cheval, quoique modifié. tion raisonnée des squelettes de plésiosaures exposés dans nos musées fait naître diverses objections d'inadéquation morphologique aux prétendues aptitudes comportementales de Nessie, compte non tenu d'une probable évolution depuis l'âge des dinosaures. Mais la grande majorité des témoins involontaires depuis des générations ne dispose que d'animaux familiers pour établir des comparaisons avec cet «animal» qui ne ressemble à rien de connu ${ }^{18}$. C'est ainsi que le portrait populaire, non scientifique, ressemble à un assemblage d'éléments hétéroclites: une tête de taureau, une crinière de cheval, des cornes retractiles d'escargot, une gueule de serpent, un cou comme une trompe d'éléphant, un dos de chameau ou d'éléphant, une queue de kangourou... Le portraitiste se devra de sélectionner, 
de compléter et d'accorder toutes ces pièces selon qu'il voudra présenter un monstre ridicule ou effrayant. Cette créature ne peut être qu'un hybride ${ }^{19}$ monstrueux gratifié de détails à valeur symbolique: des pieds griffus pour exprimer la férocité, des pattes arrière de guépard pour la vélocité, des écailles pour l'aptitude à vivre en milieu aquatique, une queue de lion pour sa noblesse de caractère, des dents de crocodile pour sa cruauté. Bref, le portraitiste tératographe est aussi un artiste.

\section{Entre folklore et littérature}

Force est de constater que l'état de nos connaissances à ce jour ne nous permet pas d'exposer un portrait exact du nessiteras rhombopteryx qui soit conforme à la réalité. Le phénomène «Nessie» échappe à la science parce qu'il ne peut pas être reproduit par l'homme de science. Ce phénomène se reproduit en toute indépendance et hors de tout contrôle humain. Il se répète à la manière d'un mythe, mais en incluant des variantes.

La description fondée sur la comparaison devient fiction. Le reporter, en publiant un article ou un ouvrage, se fait historien du présent, et parce qu'il rapporte des expériences qui ne sont pas les siennes propres - expériences qu'il synthétise, néanmoins - écrit un récit. Gilbert Durand, dans son article «Redondances mythiques et renaissances historiques» ${ }^{20}$, avance que «le choix dans la masse des événements et les tentatives de généralisation et d'interprétation» indiquent un retour au récit littéraire: "Donc l'historien est finalement un écrivain et le texte-récit est passible de toute analyse du récit...» Puisqu'il s'agit de monstre, faut-il céder à la tentation de classer ces descriptions, spontanées ou élaborées, dans le genre fantastique?

Tzvetan Todorov, dans son Introduction à la littérature fantas-

19. Claude Kappler, dans Monstres, démons et merveilles, p. 147, désigne «sous le terme général d'hybridation tous les êtres qui sont constitués d'éléments anatomiques disparates qui rompent l'aspect physique normal».

20. Champs de l'imaginaire, p. 171.

21. Introduction à la littérature fantastique, p. 29. tique, définit le concept de fantastique ${ }^{21}$ par rapport à ceux du réel et de l'imaginaire; il implique qu'au milieu d'un monde connu - dont on pourrait dire qu'il appartient au réel - un événement se produit qui ne peut s'expliquer par les lois de ce monde réel. Le témoin de l'événement doit décider que l'événement soit appartient au monde réel, soit relève d'une illusion des sens. En l'occurrence, le touriste qui regarde le Loch Ness et voit surgir à la surface une créature de taille extraordinaire doit décider qu'il voit effectivement un animal qu'il ne recon- 
22. Idem. p. 39.

23. Je me réfere à la traduction française: Dagon. Paris: Belfond, 1969, p. 5-13.

24. Par le biais du monolithe sur lequel le narrateur distingue des personnages monstrueux parce que présentant des différences par rapport à la norme.

25. Voir Claude Kappler, Monstres, Démons et Merveilles, p. 212 «Le monstre est celui dont l'aspect nous est inhabituel par la forme de son corps, sa couleur, ses mouvements, sa voix, «et même les fonctions, parties ou qualités de sa nature.» (Kappler cite La Cité de Dieu de saint Augustin); p. 215. «le monstre est un être qui s'écarte plus ou moins de la norme et tout est dans la manière dont on apprécie cet écart». naît pas ou bien qu'il est victime d'une illusion d'optique: il est surpris par la perception inopinée d'une vague, d'une loutre, d'un oiseau qu'il prend pour le fameux monstre dont on lui a tant parlé, cet animal mystérieux qui hante ce grand lac à l'eau froide et sombre. Le témoin devient personnage, ou narrateur, dans le récit du reporter-auteur qui veille à faire partager à son lecteur le désarroi du personnage. Si nous sommes dans la situation du témoin qui raconte lui-même l'événement à des amis, soit il s'efforcera d'être objectif, ce qui l'obligera à être très succinct et à s'en tenir aux faits bruts, soit il cherchera à faire partager son émotion, ce qui le placera dans la situation du reporter-écrivain.

Todorov rappelle que pour H.P. Lovecraft la qualité de fantastique pour un récit réside dans ce qu'éprouve le lecteur ${ }^{22}$. Le conte de Lovecraft intitulé Dagon ${ }^{23}$ fonctionne sur deux éléments principaux: le personnage quitte le monde réel après s'être endormi (... Le changement advint tandis que je dormais...); il en est de même pour son retour (... Quand je suis sorti des ténèbres, je me trouvais dans un hôpital...); le monde cauchemardesque dans lequel le personnage se trouve projeté (... à demi-happé par une sorte de boue d'un noir d'encre...) se transforme progressivement en un monde quasi réel, mais le personnage-narrateur veille à entretenir un sentiment de peur (... Et une satanique laideur monta des étranges royaumes des ténèbres...), d'étrangeté ${ }^{24}$ (... ils avaient une allure odieusement humaine, malgré leurs pieds palmés, leurs mains molles... Ces créatures semblaient avoir été sculptées sans souci des proportions...) et d'horreur (... Soudain, je vis la chose. Dans un léger remous au-dessus des eaux troubles, elle émergea. D'un aspect répugnant, d'une taille aussi imposante que celle de Polyphème...). L'incompréhension de l'événement inattendu anéantit la capacité de discernement du personnage-narrateur et l'accumulation précipitée de rencontres étranges engourdit celle du lecteur. Ce conte fantastique de Lovecraft illustre assez bien, en la magnifiant, ce que les témoins oculaires d'une remontée en surface intempestive de Nessie disent avoir éprouvé. Ainsi, la sensation d'horreur associée à l'impression de laideur naît de la perception de la forme ${ }^{25}$ que l'on juge différente de la norme établie.

Le phénomène du monstre du Loch Ness est devenu complexe en très peu de mois: avant l'article de l'Inverness Courier, en mai 1933, an Niseag, le cheval-des-eaux du Loch Ness était un 
être de légende doté de pouvoirs maléfiques, mais dont on pensait qu'il était la manifestation funeste d'une créature réelle; après mai 1933, an Niseag est devenu «le monstre» pour son aspect hors-norme, puis «Nessie» par dérision. Désormais, ce phénomène recouvre des aspects très divers: zoologique (est-ce un plésiosaure adapté à la niche écologique du Loch Ness après une évolution multi-millénaire?), sociologique (est-ce le fruit de l'imagination des Highlanders, et pas celle des AngloSaxons, sous l'influence du whisky?), linguistique (comment le dénommer: «an Niseag» ou «Nessie»? «monstre» ou «chevaldes-eaux»?, etc.), psychologique (celui qui prétend avoir vu le monstre souffre-t-il de frustration ou d'hallucination?) ou surnaturel (ce que certaines personnes prétendent voir serait-il la projection d'un message envoyé par Dieu ou par Satan, ou par les extra-terrestres?). Il convient de considérer le problème dans un contexte internationalisé puisqu'il a attiré à lui des chercheurs venus d'Amérique ou du Japon ${ }^{26}$. L'honnêteté intellectuelle qui semble faire défaut ici exigerait que le problème soit d'abord exposé dans sa complexité avant de choisir d'en développer un des aspects, car chacun mérite d'être analysé pour ce qu'il est. Et il faut déplorer les amalgames faciles, tel que Nessie-fantôme-soucoupe-volante-whisky, qui interdisent toute tentative d'étude approfondie.

L'incroyable, comme le bons sens, semble toutefois être la chose la mieux partagée au Royaume-uni: si Nessie n'était que le pur produit de l'imagination des indigènes de la région d'Inverness, pourquoi l'administration a-t-elle décidé, dans sa raisonnable prudence, de protéger la "chose» par décret, ainsi que le rappelle l'auteur de "The Loch Ness "Monster" », dans le numéro de Nature daté du 16 décembre 1933:

The Secretary of State for Scotland, in reply to questions, has written that he has communicated with the Chief Constable of Inverness-shire, who had already stationed five constables at different places on the loch. None has yet seen the monster; but it is guaranteed protection should it appear and be threatened.

Les autorités du district sont peut-être disposées à protéger

26. ... ces soi-disant chercheurs japonais ont conservé la réputation de fêtards peu enclins à honorer leurs notes d'hôtel.
Nessie, mais plus vraisemblablement décidées à empêcher les soi-disant chasseurs de monstres équipés de harpons et d'explosifs de mettre en danger la faune du loch et son voisinage humain. 
La question de l'identification officielle restant en suspens, on peut se demander si le produit de l'imagination engendre des illusions, dont sont victimes les témoins malgré leur sincérité, ou bien si les légendes sont nées d'observations incomplètes. Selon l'analyse de Todorov, nous hésitons à classer l'affaire Nessie dans l'étrange ou dans le merveilleux. Car dans le genre fantastique l'auteur vise à plonger son lecteur consentant dans l'hésitation entre l'étrange et le merveilleux. Qui plus est, nous pouvons remettre en cause le choix du fantastique qui allègue que le monstre du Loch Ness n'existe que dans l'imagination. L'insolite pourrait alors être le terme le plus approprié, si on lui reconnaît le sens de extra-ordinaire et d'a-normal. Il se distingue du surnaturel qui implique l'intervention de l'autre monde, qu'il soit divin ou satanique; mais saura-t-on jamais si l'exorcisme ${ }^{27}$ du Père Omand fait le 2 juin 1973 a purifié le monstre de toute malignité?

Permets que par le pouvoir accordé à Ton humble serviteur, ce Loch des Hautes Terres et les terres environnantes puissent être délivrées de tout esprit mauvais, de toute imagination vaine, de toute projection et de tout fantasme, enfin de toutes les tromperies du Malin. O Seigneur, soumets-les à la volonté de Ton serviteur, afin qu'à son ordre ils ne nuisent ni aux hommes ni aux animaux mais se retirent vers les lieux qui leur ont été assignés et qu'ils y restent à jamais.

Tout fascinant qu'il soit, le surnaturel n'offre qu'une perspective d'explication restreinte. L'insolite autorise l'auteur à chercher à surprendre son lecteur, voire à faire naître des interrogations car le possible n'est pas exclu; l'insolite permet un glissement du réel vers le fabuleux. Par ses métaphores claires aux yeux de l'initié, la fable peut guider vers l'appréhension du réel qui paraît insaisissable: le kelpie noir du Loch Ness est une

27. L'exorcisme est rapporté en détail dans la revue, Flying Saucer Review, sept.-oct.. Londres, 1973. 28. Ces légendes multiples du kelpie noir du Loch Ness illustrent les "éclairages sans cesse changeants, mais redisant toujours le même, des redondances", de G. Durand dans Redondances mythiques, in Champs de l'imaginaire, p. 179. 29. Dagon, p. 12. créature plus fabuleuse que fantastique, parce qu'il intervient dans moult récits pour servir la même morale ${ }^{28}$, contrairement au «Dagon » de Lovecraft, dont le comportement trop brièvement décrit reste incompréhensible; en effet Dagon, le dieupoisson, fait l'objet d'une seule phrase ${ }^{29}$ :

D'un aspect répugnant, d'une taille aussi imposante que celle de Polyphème, ce gigantesque monstre de cauchemar s'élança rapidement sur le monolithe, l'étreignit de ses grands bras couverts d'écailles, tandis qu'il inclinait sa tête hideuse en proférant une sorte d'incantation. 


\section{Récits et légendes}

Le roman et la nouvelle partagent une caractéristique commune qui est leur originalité ou leur unicité, différant en cela de la légende qui se répète de génération en génération. Les légendes des chevaux-des-eaux, qui vont de pair avec les légendes des taureaux-des-eaux, se retrouvent de vallée en vallée et d'île en île mais semblent avoir bien peu de chances de survivre au matérialisme du $\mathrm{XX}^{e}$ siècle: les histoires terrible de kelpie racontées l'hiver au coin du feu ont impressionné des centaines de générations d'enfants, qu'en sera-t-il des générations actuelles nourries de monstres de légendes internationales mais de valeur archétypale? Les récits ne sont guère différents des légendes d'antan, mais ils sont visuellement représentés dans les dessins animés ou les bandes dessinées. Il n'est pas assuré, comme on peut le craindre, que l'imagination des enfants se tarisse par l'imposition d'un dessin sur un récit, car les récits fantastiques, en dessins animés ou non, se trouvent multpliés et sur un même récit le jeune lecteur ou spectateur peut disposer de points de vue différents. Cela peut susciter des commentaires dans les cours de récréation et stimuler l'imagination.

Gilbert Durand, dans son ouvrage Les Structures anthropologiques de l'imaginaire, étudie le mythe du cheval. Qu'il s'agisse du cheval chtonien ou du cheval aquatique ${ }^{30}$, le cheval a été doté dans la mythologie grecque de symbolisme fort ${ }^{31}:$ «Le cheval aquatique nous semble également se réduire au cheval infernal. Non seulement parce que le même schème de mouvement est suggéré par l'eau courante, les vagues bondissantes et le rapide coursier [...] le cheval est associé à l'eau à cause du caractère terrifiant et infernal de l'abîme aquatique». La vitesse du cheval au galop est assimilée au courant de la rivière ou du torrent, la violence du cheval se compare à la vague de la mer déchaînée, mais quel est le lien entre le cheval et le lac d'Écosse sans courant où les vagues ne sont jamais phénomé-

30. Le cheval de Camargue courant en toute liberté dans les marais comme sur la lande ou les plages a fait naître les légendes du cheval blanc.

31. Les Structures anthropologiques de l'imaginaire, p. 8283. nales, mais dont la profondeur paraît abyssale tant l'eau est sombre? L'eau bondissante du torrent peut évoquer le dos du cheval en course, la vague déferlante peut rappeler la tête d'un cheval dont la crinière serait la crête chargée d'écume hérissée par le vent. Quel mouvement de l'eau d'un lac peut aider à imaginer un cheval dans un mouvement caractéristique, tel que le cheval qui s'ébroue, qui se cabre, qui rue? Peut-être faut-il 
considérer, avec G. Durand, les bruits associés au cheval ${ }^{32}$ : "C'est [...] l'aspect d'un cheval bruyant et ombrageux que le folklore, comme le mythe, imagine le tonnerre. [...] Nous retrouverons, à propos du cri animal, cet aspect bruyant de la thériomorphie. Le galop du cheval est isomorphe du rugissement léonin, du mugissement de la mer comme celui des bovidés ». Cette dernière phrase n'est pas très convaincante: le piétinement des bovidés ou des équidés en troupeau peut évoquer le grondement du tonnerre, tout comme le fracas de la mer démontée, mais il faut utiliser le fourre-tout du cri du monstre - gros animal sauvage préhistorique - pour trouver des équivalences avec le rugissement du lion ou le mugissement de la mer.

Durand rappelle ${ }^{33}$ que «les symboles bovins apparaissent comme les doublets pré-aryens de l'image du cheval». Passant en revue les mythologies d'Europe, il conclut par le constat ${ }^{34} \mathrm{de}$ «l'étroite parenté du symbolisme taurin et du symbolisme équestre». Il n'est pas utile de revenir plus longuement sur la parité relative ${ }^{35}$ de valeur du cheval-des-eaux et du taureaudes-eaux ${ }^{36}$ et l'analyse psychanalytique du cheval ou du taureau serait ici redondante, mais je soulignerai que l'angoisse dont parle Durand ${ }^{37}$ ( $« .$. une angoisse devant tout changement, devant la fuite du temps comme devant le "mauvais temps" météorologique. Cette angoisse est surdéterminée par tous les périls incidents: la mort, la guerre, l'inondation, la fuite des astres et des jours, le grondement du tonnerre et l'ouragan») ne devrait pas occulter le désir d'accaparement des

32. Ibid., p. 83-84.

33. Ibid., p. 86.

34. Ibid., p. 88.

35. Dans la mythologie celtique le cheval et le taureau n'ont rien de commun. Voir plus loin. 36. On peut noter que, dans le folklore français, les légendes de tarasques rhodaniennes, de mâchecroute lyonnaise, de drac auvergnat ou de grand'goule normande permettent de réunir toutes ces bêtes monstrueuses dont les aspects changeants les font assimiler tantôt aux chevaux, tantôt aux dragons...

37. Les Structures anthropologiques de l'imaginaire, p. 88. capacités physiques du taureau ou du cheval, comme en témoignent les centaures.

\section{Un faisceau de présomptions}

Domestiqué ou non, le cheval est un animal qui nous est familier depuis toujours, semble-t-il. Notre civilisation indoeuropéenne l'a doté de qualités observables ou supposées et en a fait une créature mythique présente dans toutes les familles ethniques et linguistiques, grecque ou latine, germanique ou celtique, qui l'ont adaptée à leur milieu géographique, ce qui justifie les variantes locales ou régionales. Les excès médiatiques dont a pâti Nessie depuis 1933 ont permis de rassembler une quantité non négligeable de légendes concernant des créa- 
tures comparables sur bien des points, à la fois morphologiques et comportementaux. Le cheval-des-eaux n'est qu'un avatar de l'archétype du cheval qui se retrouve dans la plupart des pays situés au nord du $50^{\complement}$ parallèle de l'hémisphère nord, les exceptions étant admises: ce qui inclut les Îles britanniques, la Scandinavie, la Sibérie et l'Amérique du nord. Cette fréquence remarquable de la présence de ces chevaux-des-eaux dans la zone décrite, pour s'en tenir là, a fait naître l'inévitable question que l'on ne parvient pas à éliminer: ces légendes traduisent-elles une réalité pour le moment inexplicable? Se peut-il qu'il y ait un fond de réalité derrière le cheval-anguille d'Irlande et le Nykur des îles Féroë, le Skrimsl d'Islande et le Nechtan des Celtes, Ogopogo, qui hante le lac Okanagan au Canada, et Storsjöodjuret, résidant discret du lac Storsjorn en Suède et enfin le taurisque tué par Hercule?

L'Écosse septentrionale foisonne de légendes de kelpies, et l'on rencontre au gré des récits de voyages et des mémoires des rapports parfois aussi succincts qu'inattendus, telle cette note d'un certain Richard Frank, capitaine des armées de Oliver Cromwell, «ce célèbre Loch Ness, dont on parle tant pour son île flottante...». La plus ancienne allusion connue à la présence de monstre est l'intervention de saint Columba qui refoula le monstre aquatique du Loch Ness en 565, lors de son périple missionnaire en Écosse: si l'on veut bien lui accorder le bénéfice du doute, on est en droit de s'interroger sur la récupération de cette légende locale à des fins de prosélytisme. Tous les récits qui font mention de monstres lacustres ici et là en Écosse depuis des siècles ne forment, tout au plus, qu'un faisceau de présomptions auquel il convient d'ajouter des inscriptions et des gravures.

Les cartes d'état-major mentionnent souvent, dans le nordouest des Hautes Terres, des petits lacs sans nom autre que "loch-na-beiste», lac de la bête, ce qui est une indication zoologique très vague de la part d'éditeurs dont l'humour est peu probable dans l'exercice de leur profession. Dans l'Atlas Blaens de 1635 on lit à propos du Loch Lomond cette note sybilline: «Des vagues sans vent, des poissons sans nageoires et une île qui flotte.» Suit un commentaire: «Les poissons en question n'ont pas de nageoires, ils sont une espèce de serpent, il n'y a pas à s'étonner». Les seuls serpents connus en Écosse qui vivent dans l'eau étant les anguilles, qui ne sont pas des poissons de surface, on ne peut que se perdre en conjectures. De 
même, à la Bodleian Library d'Oxford se trouve aussi une carte du Loch Tay datée de 1325-1350 sur laquelle on peut lire:In isto lacu tria mirabilia: insula natans, pisces sine intestinis, fretum sine vento, c'est-à-dire, dans ce lac il y a trois faits qui relèvent du merveilleux: une île qui nage, des poissons sans ventre, des vagues sans vent. L'île qui flotte doit faire référence à une masse assez importante qui se déplace à la surface de manière autonome, et elle peut faire penser à la photo prise par M.H. Cockrell à l'automne 1958, ou encore à la fameuse bosse de type «barque renversée » filmée par Tim Dinsdale le 23 avril 1960 pendant quatre minutes. Le poisson sans ventre ou le serpent peut décrire, de manière bien peu scientifique, le cou que certains témoins ont comparé à une trompe d'éléphant ou à un poteau télégraphique. Les vagues sans vent rappellent la photographie du sillage injustifié photographié par M.R. Lowrie le 7 août 1960 (à $16 \mathrm{~h} 15$, par temps calme) alors que celui-ci dépassait leur yacht, le Finola... Ces anciennes observations trouvant un écho surprenant dans notre siècle sont pour le moins déconcertantes.

Il reste néanmoins un ensemble de représentations à analyser: les images gravées sur des pierres par les Pictes il y a plus de mille ans. La zone géographique concernée par ces sculptures n'est pas limitée aux alentours du Loch Ness, comme F.W. Holiday ${ }^{38}$ pourrait le laisser croire, mais elle s'étend sur l'ensemble des Hautes terres. On pourrait définir trois catégories de ces représentations : les personnages humains montrant

38. F. Holiday fut le premier à introduire une relation entre le phénomène Nessie et les sculptures, ou des croquis, sur pierre. Obnubilé par tout ce qui ressemblait à un ver (orm), il ne semble pas avoir vu les kelpies et autres représentations de monstres aquatiques.

39. Claude Kappler, dans son ouvrage Monstres, démons et merveilles, p.230-

231, présente «le Triton et la Serene du Nil», le

«Diable de mer», «le Monstre marin ayant la teste d'un moyne, armé et couvert d'escailles de poisson" et "le monstre marin ressemblant à un Evesque vestu de ses habits pontificaux". surtout des guerriers à pied ou à cheval, des objets dont certaińs sont à peine identifiables, et des animaux. Ces animaux sont soit figuratifs, et l'on reconnaît aisément le cheval, le taureau, le poisson, l'aigle, le sanglier, le cerf... soit inexplicables selon nos critères scientifiques: les chevaux marins, les chameaux marins, l'éléphant aussi appelé kelpie, et d'autres que l'on ne peut que qualifier de monstres parce qu'ils ne ressemblent à rien de connu et qui, le plus souvent, sont omis dans les descriptions de pierres sculptées, méritent de figurer dans un bestiaire du moyen-âge ${ }^{39}$. L'Écosse septentrionale n'est pas un habitat attesté des chameaux et des éléphants, mais leur représentation en ces lieux peut s'expliquer par les descriptions qu'auraient pu faire les pèlerins de retour de Rome et du bassin méditerranéen. Le chameau est généralement représenté à genoux, ce qui n'a rien d'incongru; cependant, si l'on fait abstraction de la partie inférieure des sculptures, on peut s'autori- 
ser à rapprocher la tête vue de face sur le cou redressé et le dos aux bosses indistinctes à ce que certains témoins disent avoir vu à la surface du loch. L'éléphant, alias kelpie, n'est en rien comparable à l'éléphant de mer: il a une tête et un bec de cygne et sa «trompe » part du sommet du crâne (comme s'il s'agissait d'un organe érectile), il n'a pas de pattes comparables à celles des taureaux ou des sangliers, encore moins à celles des éléphants, mais ses membres antérieurs et postérieurs bien distincts du corps sont enroulés aux extrémités. Faut-il y voir un symbole ou l'incapacité de l'artiste à copier la réalité parce que non visible? Quoi qu'il en soit, l'éléphant ou kelpie par sa composition hybride, entre dans la classe des monstres.

La description physique de ces monstres dérange parce qu'on ne peut pas les classer, et il importe de ne pas les occulter. Doiton leur attribuer une quelconque valeur symbolique? Les documents transmis par les Celtes nous permettent d'affirmer que le saumon est le symbole de la sagesse, le sanglier ${ }^{40}$ est associé à l'indépendance farouche ainsi qu'au feu; mais on ne sait rien du kelpie-éléphant, ni du chameau: peut-on s'autoriser à le joindre au faisceau de présomptions, comme une "pièce à verser au dossier »? Bien qu'il ne soit pas extraordinaire de le voir traverser les lacs et les cours d'eau, le cerf, dont on trouve l'effigie fréquemment sur les pierres sculptées, n'est jamais associé au kelpie; est-ce parce qu'il est le roi des animaux dans la mythologie celtique et qu'il symbolise la fertilité sauvage ainsi que celle du chef de clan qu'il est devenu le protecteur du royaume animal?

Il faut retenir que le kelpie des légendes qui redevient toujours cheval ou taureau, apparaît souvent sous les traits d'un beau jeune homme séduisant; mais alors la belle en caressant les cheveux du séducteur y trouve des algues. Plus rarement le kelpie se déguise en superbe séductrice qui peut, par mégarde, hennir de plaisir... Nous avons déjà analysé les différences morphologiques du taureau et du cheval, il nous reste à étudier leur complémentarité symbolique dans la mythologie celtique. Le taureau, quasiment déifié, a toujours été un symbole de force, de puissance et de fertilité qui est lié au roi. Le cheval est très lié à la divinité féminine: le nom de la déesse Etain Echraidhe signifie la déesse Etain à cheval; ainsi, par effet d'assi-

40. Claude Kappler présente le sanglier marin dans Monstres, démons et merveilles, p. 229. milation, le cheval exprime à la fois la déesse équine et l'archétype de la femme-mère déifiée. Le cheval est une image complexe car il est aussi lié à la déesse de la prophétie et des 
oracles; il peut apparaître sous les traits d'une vieille femme (cailleach), plus précisément ceux de la vieille sorcière transformée en jument de la nuit (night mare) qui s'en va dispenser cauchemars et mauvais présages. Le cheval, surtout, est celui qui fait office de passeur pour l'autre-monde; c'est le nocher infernal des Celtes. La légende du kelpie entraînant dans les eaux sombres du lac sa pauvre victime frivole est en fait une parabole de la mort qui vient se saisir du simple mortel pour l'emporter au travers du miroir qu'est la surface du lac où se reflètent montagnes ensoleillées et nuages insouciants.

Ainsi le taureau, ou la vache, et le cheval sont-ils des totems complémentaires dans la mythologie celtique. Le taureau-deseaux et le cheval-des-eaux résumés en kelpie en paraissent l'ultime avatar représenté sur les blocs de pierre arborant des croix chrétiennes. La civilisation picto-celte telle qu'elle se manifeste dans le granit des Hautes Terres, ayant assimilé tour à tour le christianisme et les croyances scandinaves, nous lègue des tératoglyphes prisonniers de ses entrelacs tentaculaires. Les lectures successives des images sculptées nous entraînent vers les interprétations d'icones et vers la recherche de symboles. Tout se superpose et se complexifie: le kelpie est né du «kelp», ou varech, pour se métamorphoser en l'animal qui s'en nourrit. La magie n'ayant pas de limite, le kelpie devient tantôt l'île-qui-flotte, tantôt le poisson-sans-entrailles; le kelpie est ici vague-sans-vent et là éléphant lacustre; le kelpie se fait homme entreprenant comme le phoque se mue en sirène enjôleuse.

Le phénomène Nessie est devenu inclassable tout comme le monstre Nessie est insaisissable. Le roman ${ }^{41}$ de Frances Hendry, Quest for a Kelpie, illustre cet état de fait parce qu'il participe de la fiction et de l'histoire, du tragique et du merveilleux. Le kelpie est un ensemble hybride de fantasmes qui ne survit que

41. Quest for a Kelpie. Edinburgh: Canongate, 1986. 42. 27 juillet 1970 : le dessin est paru après que la presse eut appris qu'une expérience était tentée avec des hormones déversées dans le lac avec l'espoir d'obtenir une réaction de ces monstres trop discrets. Une autre expérience musicale fut tentée ultérieurement à l'aide d'hydrophones, sans plus de succès. dans sa fragmentation, de même que Nessie est tout à la fois le serpent de mer aux longs cils et aux lèvres pulpeuses du dessinateur du Daily Mirror ${ }^{42}$ et la masse sombre et compacte, rugueuse comme un dos d'éléphant, émergeant lentement et silencieusement tout contre la barque du garde-pêche de Fort Augustus. Pendant un nombre incalculable de générations le kelpie a provoqué l'angoisse de milliers de Highlanders, comme Nessie a excité la curiosité des uns et causé l'hilarité des autres: l'excès de crédulité des générations anciennes s'est transmise en s'amenuisant, mais ne mérite pas davantage 
43. Tels que les soldats que l'on entend les nuits de pleine lune autour du château de Urquhart.

44. Les hallucinations de F.W. Holiday ou les canulars de Compton Mackenzie.

45. Sur la rive sud du Loch Ness se trouve Boleskine House, maison étrange qui jouxte le cimetière local. Ce fut la propriété du célèbre Alistair Crowley (de 1901 à 1947) dont les pratiques maléfiques inquiétaient le voisinage. 46. Dans la propriété de M. Gordon Macintosh, au lieu dit Tychat, près de Drumnadrochit, rive nord, on peut voir le cercle magique de sorbiers dans une étrange petite clairière...

47. Où classer la théorie scientifique, ou pseudoscientifique, selon laquelle ce que les témoins de bonne foi disent avoir vu sur le lac ne serait que la restitution accidentelle par désagrégation de la roche d'ondes captées par la même roche au passage de plésiosaures il y a des millions d'années: sorte de rediffusion télévisuelle? 48. Ce qui signifie littéralement le «lac des ténèbres". notre mépris que le tapage démesuré de notre presse incrédule. Et la maladresse sincère de centaines de témoins éberlués vaut bien la manipulation malveillante de ces dizaines d'exploiteurs avides.

Si l'on s'interroge sur le rapport entre le kelpie et le surnaturel, on constate d'abord que le nom de «kelpie» est différent de "cheval-des-eaux", même si les deux termes recouvrent des valeurs très proches, et que cela indique que leurs fonctions respectives diffèrent. Le cheval-des-eaux décrit une réalité supposée qui n'est pas prouvée, du moins de manière convaincante. Le «kelpie», en assumant l'ensemble des variantes de légendes, tient le rôle de passeur entre le naturel énoncé dans le nom de nessiteras rhombopteryx et le surnaturel surgissant dans le cheval maléfique parfois anthropomorphe. On est alors en droit de penser que le surnaturel se manifeste par l'intervention de l'homme qui, par peur (conjurée par l'aspect donné à la manifestation de l'angoisse devant la souffrance et la mort) ou par jeu (concrétisé par la dérision ou l'extravagance, et masquant spontanément l'ignorance), opère des rapprochements incongrus entre un phénomène jusqu'ici inexpliqué et d'autres manifestations du surnaturel sur les abords du Loch Ness : fantômes ${ }^{43}$, extraterrestres ${ }^{44}$, exorcisme et magie noire ${ }^{45}$, lutins ${ }^{46}$ ou illusion ${ }^{47}$. Le foisonnement de bizarreries émousse notre crédulité. Mais s'il est une certitude, c'est bien celle que le kelpie héberge nos émotions dont, par pudeur, on aime cacher le caractère naturel; il fait diversion. Bien honnête celui qui osera avouer le frisson qu'il a ressenti en écoutant chanter la Complainte du Cheval-des-eaux sur les bords du Loch na Dubhrachan ${ }^{48}$ de l'île de Skye: une belle demoiselle de Skye malencontreusement s'éprit d'un kelpie qui lui était apparu sous les traits d'un beau jeune homme; ils se marièrent et vécurent des jours heureux jusqu'à l'instant où elle s'aperçut de l'épouvantable tromperie, car le fourbe avait du sable sur la poitrine et des algues dans les cheveux; elle parvint à s'enfuir, laissant tout derrière elle pour échapper à son funeste destin. Mais elle finit ses jours dans la hantise de l'horreur. 


\section{Bibliographie}

Bauer, Henry. The Loch Ness "Monster» - a Bibliography. Blacksburgh, VA: Virginia Polytechnic Institute \& State University, 1982. (78p.)

Durand, Gilbert. Champs de l'imaginaire. Grenoble: Ellug, 1996.

—, Les Structures anthropologiques de l'imaginaire. Paris: Dunod, 1992.

Gray, Tom \& Ferguson Lesley. Photographing Carved Stones. Balgavies: The Pinkfoot Press, 1997.

Harrison, Paul. The Encyclopaedia of the Loch Ness Monster. Londres: Hale, 1999.

Hendry, Frances. Quest for a Kelpie. Edinburgh : Canongate, 1986.

Holiday, F.W. The Great Orm of Loch Ness - A Practical Inquiry into the Nature and Habits of Water-Monsters. Londres: Faber, 1968.

Kappler, Claude. Monstres démons et merveilles à la fin du Moyen Âge. Paris: Payot, 1980.

Lovecraft, H.P. Dagon. Paris : Belfond, “J'ai lu”, 1969.

McDonald, Lorraine. Celtic Totem Animals. Brodick: Clan Dalriada, 1992.

Mackensie, Compton. The Rival Monster. London: Chatto and Windus, 1952.

Ritchie, Anna. Picts. Edinburgh: Historic Scotland, 1999.

Todorov, Tzvetan. Introduction à la littérature fantastique. Paris : Le Seuil, 1970.

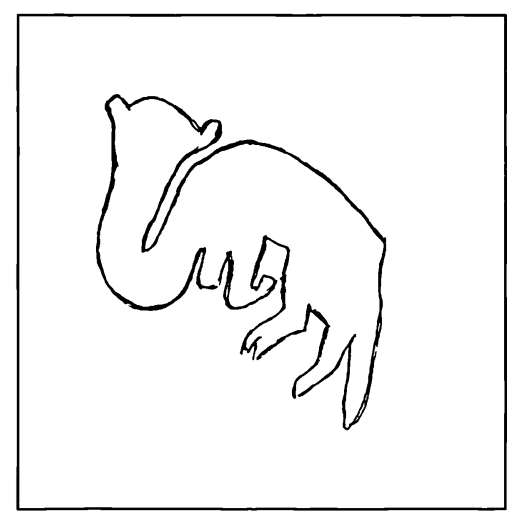

1. Le chameau-des-eaux tel qu'il apparaît sur une pierre dressée exposée au musée de Meigle (Perthshire) parmi d'autres animaux et personnages. Il n'y pas d'explication autre que " animal exotique»... (Croquis J. Berton).

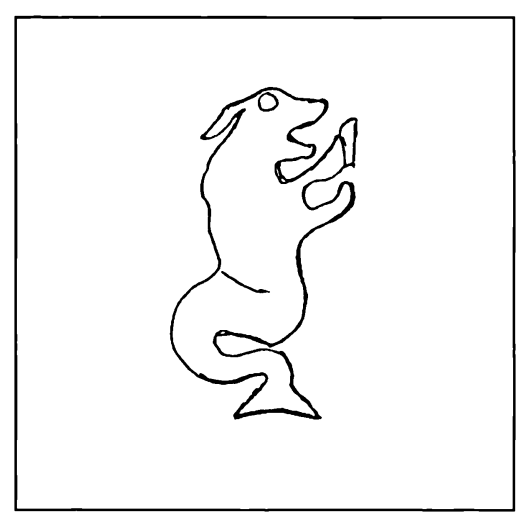

2. À défaut de nom, je le qualifie de "chien-des-eaux»: la partie antérieure pourrait représenter un chien, la partie inférieure en queue de sirène se termine par une queue de poisson... Ce monstre a été sculpté sur une pierre rectangulaire arborant une croix. Musée de Dunfallandy, Perth. (Croquis J. Berton) 


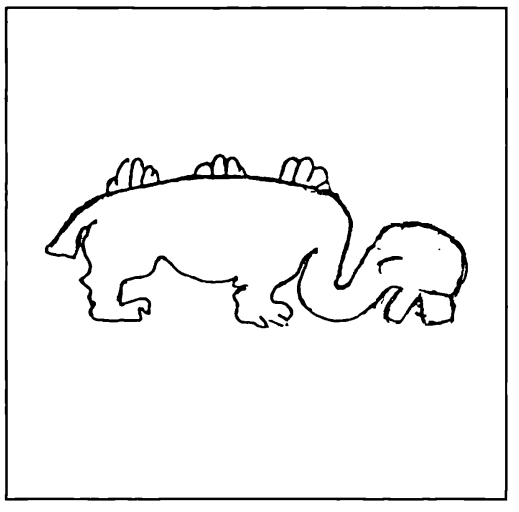

3. Créature non identifiée apparaissant dans une procession d'animaux de part et d'autre de la croix sculptée sur une pierre rectangulaire exposée à

$S t$ Vigeans (près de Arbroath). Est-ce une autre représen-

tation du "chameau-des-eaux" ou un dinosaure

quelconque à cause des bosses ou nageoires dorsales? (Croquis J. Berton).

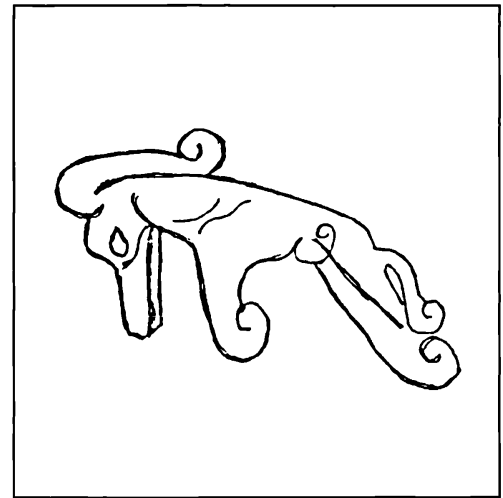

4. Le "kelpie», aussi appelé "éléphant", tel qu'il apparaît sur de nombreuses pierres. Au musée de Meigle, Perthshire, on peut en voir plusieurs. (Croquis J. Berton)

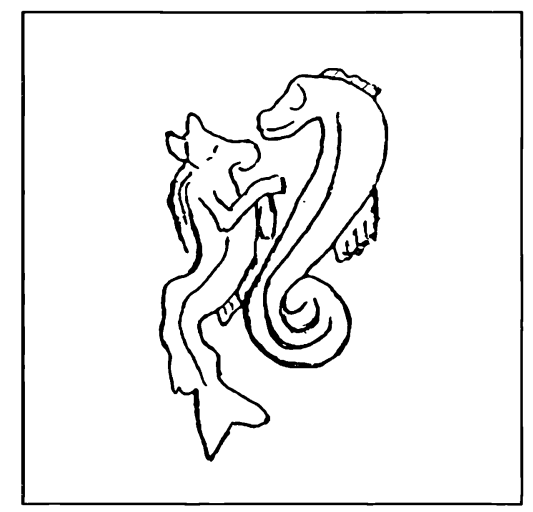

5. Cheval marin et hippocampe. Ces deux créatures apparaissent sur une pierre dressée exposée au musée de Meigle, Perthshire. (Croquis J. Berton). 